山्山FANÇAISE

$>\mathrm{DE}$

$\stackrel{1=1}{\simeq}$ PÉDAGOGIE
Revue française de pédagogie

Recherches en éducation

$196 \mid 2016$

Apprendre à lire et à écrire au cours préparatoire : enseignements d'une recherche collective

\title{
Influence de la planification de l'étude du code alphabétique sur les performances des élèves en décodage au cours préparatoire
}

Effects of phonics instruction on first graders progress on the decoding task

Jérôme Riou et Valérie Fontanieu

\section{OpenEdition}

\section{Journals}

Édition électronique

URL : https://journals.openedition.org/rfp/5073

DOI : $10.4000 / \mathrm{rfp} .5073$

ISSN : 2105-2913

Éditeur

ENS Éditions

Édition imprimée

Date de publication : 30 septembre 2016

Pagination : 49-66

ISSN : 0556-7807

Référence électronique

Jérôme Riou et Valérie Fontanieu, «Influence de la planification de l'étude du code alphabétique sur les performances des élèves en décodage au cours préparatoire », Revue française de pédagogie [En ligne], 196 | 2016, mis en ligne le 30 septembre 2019, consulté le 27 janvier 2022. URL : http:// journals.openedition.org/rfp/5073; DOI : https://doi.org/10.4000/rfp.5073 


\title{
Influence de la planification de l'étude du code alphabétique sur les performances des élèves en décodage au cours préparatoire
}

\author{
Jérôme Riou \\ Valérie Fontanieu
}

\begin{abstract}
Quelle est l'influence de la planification de l'étude du code alphabétique et du choix des textes supports d'enseignement de la lecture sur les performances des élèves en décodage au cours préparatoire (première année primaire)? Pour répondre à cette question, les auteurs s'appuient sur l'observation des pratiques pédagogiques de 131 enseignants expérimentés et sur l'évaluation des progrès des élèves réalisées dans le cadre de l'enquête Lire et Écrire. Ils soulignent l'influence significative de deux caractéristiques des pratiques d'enseignement sur l'apprentissage du décodage, d'une part la vitesse d'étude explicite des correspondances graphophonémiques au début du cours préparatoire et d'autre part la proportion de graphèmes déchiffrables dans les textes choisis comme supports de leçons de lecture. Les modélisations statistiques multiniveaux portant sur un échantillon de 2507 élèves leur permettent d'identifier des inférences causales en contrôlant les caractéristiques des élèves, des enseignants et des classes.
\end{abstract}

Mots-clés (TESE) : lecture, école primaire, enseignement, planification, efficacité

\section{Questions de recherche}

En 2000, à la demande du Congrès des États-Unis, une méta-analyse du National Institute of Child Health and Human Development (100000 travaux inventoriés) a été consacrée à l'enseignement et aux premiers apprentissages de la lecture (National Reading Panel [NRP], 2000). En conclusion, ses auteurs ont indiqué la supé- riorité des démarches dans lesquelles les correspondances graphophonémiques étaient systématiquement enseignées sur celles dans lesquelles ces correspondances ne l'étaient pas ou l'étaient peu. Autrement dit, ils ont souligné l'intérêt d'un enseignement explicite et précoce des correspondances entre phonèmes et graphèmes, c'est-à-dire entre les unités sonores élémentaires du langage parlé et les lettres ou groupes 
de lettres qui leur correspondent dans une langue alphabétique. En revanche, ils n'ont pas noté de différence significative entre «l'approche synthétique qui consiste à apprendre aux élèves à associer une lettre isolée (ou une combinaison de lettres) au son correspondant, puis associer les sons pour former des mots » ou «l'approche analytique dans laquelle les élèves apprennent d'abord des syllabes complètes, puis les correspondances lettres-sons dans ces syllabes » (NRP, 2000, chapitre 2, p. 93, traduit par nous).

Aucune étude réalisée auprès d'enfants francophones n'a été retenue par les chercheurs du NRP car aucune ne correspondait à leurs critères de scientificité. Toutefois, les résultats de la méta-analyse américaine et bien d'autres qui allaient dans le même sens (Goigoux, 1993a, 1993b, 2000; Braibant \& Gérard, 1996; Gombert, Colé, Valdois et al., 2000) furent pris en compte dans les programmes de l'école française arrêtés en 2002. Ceux-ci enjoignaient aux professeurs d'aider les élèves à mémoriser les relations entre les graphèmes et les phonèmes, de leur permettre de structurer ces informations de manière rigoureuse, de les réviser fréquemment, et de viser de manière systématique «la mémorisation des principaux assemblages syllabiques entre voyelles et consonnes dans les différentes combinaisons possibles» (MEN, 2002, p.44). Ils précisaient que cet apprentissage reposait aussi sur «l'analyse de mots entiers en unités plus petites référées à des connaissances déjà acquises » (MEN, 2002), par exemple en utilisant des analogies syllabiques (utiliser le «pa» de «papa» pour segmenter et décoder «Paris», etc.). Autrement dit, les activités de synthèse et d'analyse étaient jugées complémentaires pour l'enseignement de la lecture et de l'écriture et les maîtres étaient incités à les conduire de front.

Pour orienter ses prescriptions ou ses recommandations, le ministère de l'Éducation nationale ne disposait pas d'étude francophone portant sur l'efficacité d'un enseignement précoce et explicite des correspondances graphophonémiques. II ne disposait pas non plus de comparaisons sur l'efficacité respective des approches didactiques qualifiées de graphémiques (basées sur l'étude de la valeur sonore des lettres) ou de phonémiques (basées sur l'étude de la transcription des sons), c'est-à-dire de celles qui privilégient le décodage (des lettres aux sons) ou l'encodage (des sons aux lettres). Par conséquent, la question de la planification de l'étude du code alphabétique, autrement dit de la vitesse d'enseignement des correspondances graphophonémiques et tée, notamment en langue française, et elle l'est restée depuis (Nonnon \& Goigoux, 2007; Feyfant \& Gaussel, 2007; Cusset, 2014; Ecalle \& Magnan, 2015). Ce constat de carence, déjà dressé par le jury de la conférence de consensus sur la lecture (PIREF, 2003), a conduit le ministère de l'Éducation nationale à financer deux nouvelles recherches de grande envergure considérant que les politiques publiques et les pratiques professionnelles devaient être fondées sur les meilleures preuves scientifiques possibles (voir Saussez \& Lessard, 2009, pour une première synthèse francophone de l'approche dite de «l'éducation basée sur la preuve»).

La première recherche a été réalisée en 2010-2011 dans des zones d'éducation prioritaire de la région lyonnaise à l'aide d'une méthodologie expérimentale. L'équipe du psychologue Édouard Gentaz a comparé les progrès de deux groupes d'élèves : un groupe expérimental auquel on a proposé un programme combinant des entraînements centrés sur le code et la compréhension ainsi qu'une progression de l'enseignement des correspondances graphèmes-phonèmes établie par Liliane Sprenger-Charolles (Dehaene, 2011), et un groupe témoin qui a bénéficié des pratiques habituelles à dominante phonémique. Au terme de cette expérimentation, au cours de laquelle les enseignants des deux groupes continuaient d'utiliser leur manuel de lecture, aucune différence d'efficacité n'a été relevée (Dehaene, 2011, p.109-110; Gentaz, Sprenger-Charolles, Colé et al., 2013).

La seconde recherche a été initiée par Roland Goigoux en 2012 dans une perspective plus écologique, c'est-à-dire visant à évaluer l'efficacité des pratiques habituelles des maîtres de cours préparatoire sans qu'un dispositif expérimental vise à les transformer (voir l'article de Jarlégan, Piquée, Fontanieu et Goigoux dans ce numéro). Cette recherche relève du paradigme de I'Educational Effectiveness Research (Van Damme, Opdeakker, Van Landeghem et al., 2009) élaboré en sciences de l'éducation dans le droit fil des travaux sur «l'effet-maître » à l'école élémentaire (voir Attali \& Bressoux, 2002, ou Bressoux, 2011, pour des synthèses). L'équipe pluridisciplinaire de recherche postulait qu'il est possible de comparer le fonctionnement de pratiques d'enseignement diversifiées et d'apprendre de la variété ainsi décrite (Duru-Bellat \& Mingat, 1998). Elle a donc organisé une enquête sur un vaste échantillon de classes proposant différentes options didactiques (Goigoux, Jarlégan \& Piquée, 2015).

C'est dans le cadre de ce programme de recherche que nous avons entrepris d'étudier l'influence de la 
planification de l'étude du code alphabétique. Nous souhaitions savoir si nous retrouverions dans l'école française les résultats établis en langue anglaise, notamment quant à la pertinence d'un enseignement explicite et précoce des correspondances entre graphèmes et phonèmes. Nous souhaitions également savoir si les approches graphémiques et synthétiques étaient plus efficaces que les approches phonémiques et analytiques ou mixtes. Nous voulions savoir enfin si la programmation de l'étude du code (la vitesse d'enseignement des correspondances graphophonémiques et le choix des correspondances) avait une influence sur la qualité des apprentissages, et nous nous interrogions sur les effets différenciés de l'étude explicite des correspondances graphèmes-phonèmes selon le niveau de compétence des élèves en code à l'entrée du cours préparatoire.

Comme Goigoux (2000) ou Sprenger-Charolles et Colé (2006), nous pensions que les élèves apprennent mieux à lire et à écrire s'ils acquièrent sans tarder des compétences à déchiffrer qui éveillent leur curiosité pour le fonctionnement du code alphabétique et qui facilitent leur auto-apprentissage (Share, 1995), tout en évitant découragements et tâtonnements hasardeux (voir aussi sur ce point Downing \& Fijalkow, 1984 ou Chauveau \& Rogovas-Chauveau, 1989). C'est pourquoi nous voulions tester I'hypothèse selon laquelle les enseignants qui conduisent leurs leçons de lecture sur des écrits (phrases, textes) en grande partie déchiffrables seraient plus efficaces que ceux qui confrontent les élèves à des écrits peu déchiffrables. Deux critères didactiques pouvaient concourir à fonder ces différences : d'une part le nombre et la fréquence des correspondances phonographiques étudiées préalablement à la lecture de l'écrit et d'autre part les caractéristiques linguistiques de cet écrit selon que l'enseignant choisissait ou non des textes composés de graphèmes préalablement étudiés.

L'équipe de Gentaz avait formulé l'hypothèse selon laquelle l'enseignement des correspondances graphophonémiques est d'autant plus efficace qu'il débute par l'étude de graphèmes fréquents en français mais aussi de ceux dont la correspondance avec un phonème est régulière ou dont la prononciation isolée est aisée. Une planification de ce type a été élaborée par Liliane Sprenger-Charolles (Dehaene, 2011, p. 119-130) à partir des caractéristiques distributionnelles des associations graphèmes-phonèmes des unités infralexicales et lexicales dans un corpus issu de 54 manuels scolaires en usage dans le primaire, du CP au CM2 (Manulex-infra;
Peereman, Lété \& Sprenger-Charolles, 2007). Nous voulions savoir dans quelle mesure les enseignants de cours préparatoire choisissaient des planifications répondant à de tels critères de rationalité et quelles étaient les conséquences de ces choix sur les apprentissages des élèves. C'est pourquoi nous avons relevé les écrits utilisés dans 131 classes et avons analysé leur composition en les comparant aux graphèmes explicitement étudiés par les enseignants. Nous avons choisi pour cela une semaine test, la dixième de l'année, après avoir inventorié les correspondances graphophonémiques enseignées au cours des neuf semaines précédentes, opérationnalisant par la même occasion le critère de précocité.

En faisant porter notre enquête sur le début de I'année scolaire (neuf premières semaines) nous postulions :

- d'une part, que les choix initiaux des maîtres étaient révélateurs de choix de planification qui allaient se confirmer durant le reste de l'année (la structuration des premières activités d'enseignement pèse sur les activités ultérieures);

- d'autre part, que ces choix influent fortement et durablement sur la qualité des apprentissages des élèves (NRP, 2000; INSERM, 2007).

- En résumé, après avoir présenté notre méthodologie de recherche, nous exposerons nos principaux résultats en réponse à trois questions :

- le nombre et la nature des correspondances graphophonémiques explicitement enseignées au début de l'année (neuf premières semaines) ont-ils un effet sur les performances finales des élèves en décodage?

- les élèves progressent-ils davantage selon que l'entrée privilégiée pour l'étude des correspondances est graphémique ou phonémique?

- la part déchiffrable des textes utilisés comme supports d'enseignement de la lecture influence-t-elle positivement les performances des élèves en décodage?

\section{Méthodologie}

\section{L'évaluation des compétences des élèves en décodage}

À l'entrée du cours préparatoire, la mesure des performances des élèves en décodage a reposé sur quatre épreuves. La première est extraite d'EVALEC, une batterie informatisée d'évaluation diagnostique des 
troubles spécifiques d'apprentissage de la lecture'1 (Sprenger-Charolles, Colé, Béchennec et al., 2005). Elle consiste à supprimer la première syllabe de 10 pseudo-mots énoncés par l'évaluateur ${ }^{2}$ (trisyllabiques de la forme (VCVCV), puis le premier phonème de 24 syllabes (12 syllabes de la forme CVC et 12 syllabes de la forme CCV). Le pourcentage d'erreurs et la durée totale de passation de l'épreuve (hors entraînements) ont été calculés. La seconde évalue la connaissance du nom des lettres de l'alphabet. La troisième et la quatrième sont des épreuves chronométrées empruntées aux études de Gentaz, Sprenger-Charolles, Colé et alii $(2013,2015)$. Elles correspondent à la lecture de mots familiers et de pseudo-mots dont certains comportent un graphème contextuel, autrement dit un graphème pouvant prendre différentes valeurs selon les lettres qui l'entourent.

À la fin du cours préparatoire, les épreuves d'analyse phonologique et de lecture ont été reprises à l'identique, celle de connaissance du nom des lettres a laissé place à une épreuve de fluence qui mesure la vitesse de déchiffrage des mots en contexte (épreuve tirée d'OURA-LEC/CP Enseignants ${ }^{3}$ ).

Les scores des quatre épreuves initiales ont été centrés et réduits puis additionnés pour le calcul d'un score unique à l'entrée du cours préparatoire (codez), il en est de même avec les scores des épreuves finales (codefz). Dans les modèles statistiques multiniveaux, les scores codez et codefz ont permis d'évaluer les effets des variables didactiques sur les performances des élèves en décodage.

\section{Analyses multiniveaux}

La construction des modèles multiniveaux suit les étapes suivantes (Bressoux, 2010). Tout d'abord, un modèle dit vide (modèle 0 ) n'incluant aucune variable explicative fournit une décomposition de la variance totale des performances finales des élèves en code en une variance interclasses et une variance intraclasse, ainsi qu'une estimation de la part de variance attri-

1 Extrait du guide : "Pour les tests d'analyse phonologique et de mémoire, EVALEC n'utilise que des pseudomots afin d'éviter les biais liés à des différences de vocabulaire».

2 À noter que contrairement au test original informatisé, les items n'ont pas été enregistrés, ce qui aurait pu éviter les biais dus à la qualité de l'articulation des évaluateurs et au recours à des aides labiales (qui peuvent faciliter principalement la tâche syllabique). buable aux classes. Dans le modèle 1, l'introduction des performances initiales des élèves (variable de contrôle de niveau 1) permet en outre d'estimer le pouvoir explicatif du score initial ( $33 \%$ de la variance interclasses et $40 \%$ de la variance intraclasse). Puis, les caractéristiques des élèves sont introduites comme variables de contrôle de niveau 1 (modèle 2 ) et les caractéristiques des enseignants et des classes comme variables de contrôle de niveau 2 (modèle 3). Ensuite, différentes formes d'effets (effets linéaires moyens, formes quadratiques, effets d'interaction avec le score initial et effets paliers) sont recherchées pour chacune des variables didactiques. Pour l'étude d'effets paliers, les pratiques de classe sont catégorisées selon différents découpages : en fonction des frontières constituées par - 1 et + 1 écart-type ( 3 catégories de classes), en fonction des tiers ( 3 catégories de classes en nombre équitable) et des quartiles (4 catégories de classes en nombre équitable). Selon les résultats des analyses, les frontières sont affinées par tâtonnements successifs.

Les analyses statistiques ont porté sur l'ensemble de l'échantillon, puis sur trois groupes d'élèves constitués selon leur niveau initial en code. Les élèves faibles en code qui obtenaient des scores inférieurs ou égaux à $-1 / 2$ écart-type (706 élèves) au début du cours préparatoire, les élèves forts en code qui obtenaient des scores supérieurs ou égaux à $+1 / 2$ écart-type (656 élèves), et les élèves aux scores intermédiaires compris entre $-1 / 2$ et $+1 / 2$ écart-type (1 145 élèves). Dans les trois cas, les 131 classes étaient représentées.

\section{Établir une liste de correspondances graphophonémiques}

Afin de répondre à nos questions de recherche, nous avions besoin de dresser l'inventaire des correspondances graphophonémiques explicitement enseignées dans chacune des classes de l'étude pendant les neuf premières semaines de l'année scolaire. Le trop grand nombre de correspondances (le système orthographique du français comporte 130 graphèmes pour 36 phonèmes) nous a obligés à procéder à une réduction afin que l'outil de recueil des données soit maniable et fiable. Nous avons donc choisi de ne conserver que les graphèmes les plus fréquents relevés dans les manuels scolaires inventoriés dans la base Manulex (Lété, Sprenger-Charolles \& Colé, 2004; Ortéga \& Lété, 2010) et les correspondances graphophonémiques habituellement étudiées au cours préparatoire en recoupant les tables des matières d'une 
dizaine de manuels. La liste que nous avons élaborée comporte 63 graphèmes associés à leur(s) correspondant(s) phonémique(s) et 12 graphèmes muets. Nous avons parfois regroupé des graphèmes voisins correspondant au même phonème, par exemple la lettre «a » associée à la lettre «à » accentuée, ou bien le graphème «ain » associé au graphème «aim» (voir annexe 1).

\section{La précocité de l'étude explicite des correspondances graphophonémiques et l'entrée choisie pour les enseigner}

Notre première question de recherche concerne la précocité de l'étude explicite des correspondances graphophonémiques. Pour y répondre, nous avons créé un tableau à partir de la liste de correspondances précédemment établie. Ce tableau permet le relevé de deux types d'indicateurs : d'une part, le numéro de la semaine qui correspond à l'enseignement explicite d'une correspondance et d'autre part, l'entrée choisie par l'enseignant pour l'étudier. Nous avons considéré que l'étude était explicite lorsque la correspondance avait fait l'objet d'une institutionnalisation et que les élèves disposaient d'une référence écrite permanente individuelle ou collective à laquelle ils avaient accès. Nous avons notamment distingué les correspondances explicitement enseignées de simples mises en relation orales entre les graphèmes et les phonèmes ou de mises en relation écrites éphémères. Les enquêteurs se sont rendus à deux reprises (fin septembre et fin octobre) dans les classes dont ils avaient la charge afin de relever, avec l'aide des enseignants, les traces de l'enseignement dispensé dans les cahiers des élèves, dans leur manuel et/ou sur les affichages muraux. Ils ont indiqué, en face de chaque correspondance institutionnalisée, le ou les types d'aide-mémoire proposés aux élèves parmi 8 possibilités ${ }^{4}$. Notons au passage que

4 Les 8 items de l'aide-mémoire sont les suivants : 1) Affichage éphémère des correspondances graphèmes-phonèmes (CGP) au tableau effaçable (durée : quelques minutes ou heures; durée inférieure à la semaine) ; 2) Affichage mural permanent des CGP (affichettes ajoutées au fur et à mesure de l'étude, tableaux récapitulatifs...) (durée de la présence de l'affichage : au moins une semaine, mais elle peut s'étendre sur une période, un trimestre, une année); 3) Cahier ou classeur de l'élève (CGP dans le cahier du jour, cahier de son, cahier d'écriture, cahier de devoirs du soir...); 4) Manuel (avec mention explicite des (GP); 5) Fichier (avec mention explicite des (GP); 6) Autre dispositif d'aide-mémoire : représentation des gestes Borel-Maisonny, des personnages de la Planète des alphas, des onomatopées associées à des historiettes, etc.; 7) Affichage mural permanent des textes supports dans lesquels sont indiqués les graphèmes étudiés; 8) Autre (préciser quoi dans la colonne remarque). la totalité des correspondances enseignées dans les 131 classes de l'étude font partie de la liste restrictive que nous avions établie. Par ailleurs, les enquêteurs n'ont pas éprouvé de difficulté à renseigner le tableau de relevé d'étude du code, ce qui témoigne de sa fonctionnalité et de la fiabilité du recueil. Le nombre de correspondances explicitement enseignées au cours des neuf premières semaines de classe constitue un indicateur de précocité que nous avons nommé tempo. Ainsi, nous avons testé statistiquement l'influence de la variable didactique tempo sur les performances des élèves en décodage.

La seconde question de recherche porte sur l'entrée choisie par les enseignants pour aborder l'étude des correspondances graphophonémiques. Cette étude peut débuter par le phonème $(P)$ qui sera ensuite transcrit, ou bien par le graphème dont la valeur sonore sera recherchée (G), ou encore en combinant les deux (PG) et en les introduisant simultanément. Pour chacune des correspondances institutionnalisées au cours des neuf premières semaines de classe, l'enquêteur a reporté l'une des entrées P, G ou PG dans le tableau de relevé de l'étude du code. Selon l'entrée à laquelle l'enseignant a eu prioritairement recours lorsqu'il étudiait explicitement une nouvelle correspondance, il a été classé dans l'une des trois catégories suivantes : entrée graphémique (si G > $70 \%$ ), entrée phonémique (si $\mathrm{P}>70 \%$ ) ou entrée mixte (si $\mathrm{P}<70 \%$ et $\mathrm{G}<70 \%$ ). Nous avons ainsi constitué une variable catégorielle à trois modalités nommée entreecgp. Puis, nous avons testé l'influence de cette variable sur les performances des élèves en décodage.

\section{Les textes supports de lecture}

La troisième question de recherche porte sur le pourcentage de graphèmes déchiffrables par les élèves lorsqu'ils sont confrontés à des textes de lecture, dans des situations de classe habituellement nommées «découverte de texte». Afin de calculer ce pourcentage ${ }^{5}$, que nous appellerons désormais rendement effectif, nous avons recueilli l'ensemble des textes qui ont servi de supports de lecture au cours de la dixième semaine de classe. Un à un, nous les avons segmentés en graphèmes

5 Ce pourcentage a été calculé sans les correspondances graphophonémiques institutionnalisées au cours de la dixième semaine de classe car nous ne savions pas si l'étude explicite de ces correspondances avait précédé ou non la situation de découverte de texte. 
puis nous avons pointé ceux qui avaient été explicitement étudiés en classe en consultant la liste des correspondances graphophonémiques et des graphèmes muets enseignés. Nous nous sommes assurés qu'aucune correspondance n'était hors liste puis nous avons calculé la proportion de graphèmes déchiffrables par des élèves qui connaîtraient tout ce qui a été étudié et seulement cela. Nous avons ainsi créé une troisième variable didactique dont nous pouvons évaluer les effets sur les performances des élèves en décodage.

Pour les raisons que nous avons indiquées plus haut, le tableau de relevé de l'étude du code ne comportait pas toutes les correspondances graphophonémiques et tous les graphèmes muets existant en français. Nous avons procédé à des ajouts de deux types pour analyser le corpus de textes recueillis et calculer les rendements effectifs : les uns répondaient à une exigence d'exhaustivité, les autres à une exigence de précision des découpages graphémiques. Les correspondances graphophonémiques et les graphèmes muets que nous avons ajoutés correspondent à des inconsistances de l'orthographe ou sont rares en français ${ }^{6}$. Les graphèmes muets porteurs de marque verbale ont dû être précisés. Par exemple, le verbe «crient » est composé des phonogrammes $\|C »\| r$,$» , et «i » qui trans-$ crivent les phonèmes $/ \mathrm{k} /$, /R/ et $/ \mathrm{i} /$, et du morphogramme "ent» qui marque la troisième personne du pluriel des verbes du premier groupe. Mais le verbe «aiment» est composé des phonogrammes «ai » et « $m$ » qui codent les phonèmes / $E$ / et $/ \mathrm{m} /$, de la lettre «e» diacritique qui influe sur la prononciation des graphèmes « $m$ » et «ai», et du morphogramme «nt» qui marque la troisième personne du pluriel des verbes du premier groupe (Catach, 2012). Autre exemple, les lettres «e» et «s» forment un seul graphème dans le verbe «cries» mais deux graphèmes distincts dans le

6 Nous avons ajouté les lignes de la lettre «a» prononcée /E/ dans rayure ou /e/ dans effrayant, des graphèmes "ai », "ay », "aî » et de leurs correspondants phonémiques /e/, /e/, /E/, de la lettre «c» prononcée /g/ dans seconde, de la lettre «g» de Gina, de la lettre «o» prononcée /WA/ dans «voyage», du graphème "on » et de son correspondant phonémique /e/ dans "monsieur», du graphème «oi » et de son correspondant phonémique /o/ dans «oignon », de la lettre «u » prononcée /œ/ dans «hum» et /w/ dans "aquarium», et de la lettre «z»prononcée /d/ dans «pizza», qui correspondent à des inconsistances de l'orthographe. Nous avons aussi ajouté celles des graphèmes «ë», «ï», «î̀, «oî», «ouin», «où», «SC》, « Û » et de leurs correspondants phonémiques respectifs / $E /$, /j/ (yod), /i/, /wa/, /w\%/, /u/, /s/ et /y/, qui sont peu fréquents en français. Enfin, nous avons ajouté les lignes des lettres « $m$ » et « $r$ » qui sont muettes dans les mots "automne» et "monsieur», ainsi verbe «chantes». Dans ce dernier, la lettre «e» porte une valeur diacritique puisqu'elle influe sur la prononciation de la lettre « $t$ », et la lettre «s $s$ porte la marque de la deuxième personne du singulier des verbes conjugués. Autrement dit, lorsque la lettre «e » n'était pas diacritique, nous avons considéré les formes verbales «ent» et "es» comme un seul graphème. En revanche, lorsque la lettre «e» était diacritique, nous l'avons considérée comme un graphème.

Pour résumer, la grille de référence que nous avons élaborée pour segmenter les textes supports de lecture comporte 98 lignes. Elle est composée de 81 lignes de correspondances graphophonémiques et de 17 lignes de graphèmes muets. Cette grille nous a permis d'identifier l'ensemble des graphèmes des textes proposés aux élèves dix semaines après la rentrée scolaire, de les comparer à ceux qui avaient été explicitement étudiés et de calculer le rendement effectif de chacune des classes de l'enquête Lire et Écrire.

\section{Résultats}

Dans les paragraphes suivants, nous décrirons les variables didactiques tempo, entreecgp et rendement effectif et nous évaluerons leur influence sur les performances des élèves en décodage.

\section{Variable didactique tempo}

\section{Résultats descriptifs}

Les tempos sont très différents d'une classe à l'autre et s'échelonnent de 5 à 26. Autrement dit, au cours des neuf premières semaines de l'année scolaire, certaines classes étudient explicitement 5 correspondances graphophonémiques alors que d'autres en étudient plus de 20. Le tempo moyen est de $11,4\left(\sigma^{7}=4,2\right)$ et la médiane se situe à 10 .

86 enseignants sur 131 s'appuient sur un tempo compris entre -1 et +1 écart-type, soit entre 8 et 15 . Toutefois, 21 enseignants conduisent les apprentissages sur des tempos plus lents, et 24 privilégient des tempos rapides de 16 correspondances et plus. La distribution ordonnée par tranches de déciles montre une croissance progressive des tempos moyens jusqu'au neuvième décile, puis une rupture entre le neuvième et le dixième décile, les enseignants du dernier décile étudiant entre 17 et 26 correspondances (voir figure 1).

$7 \quad \sigma$ signifie écart-type. 


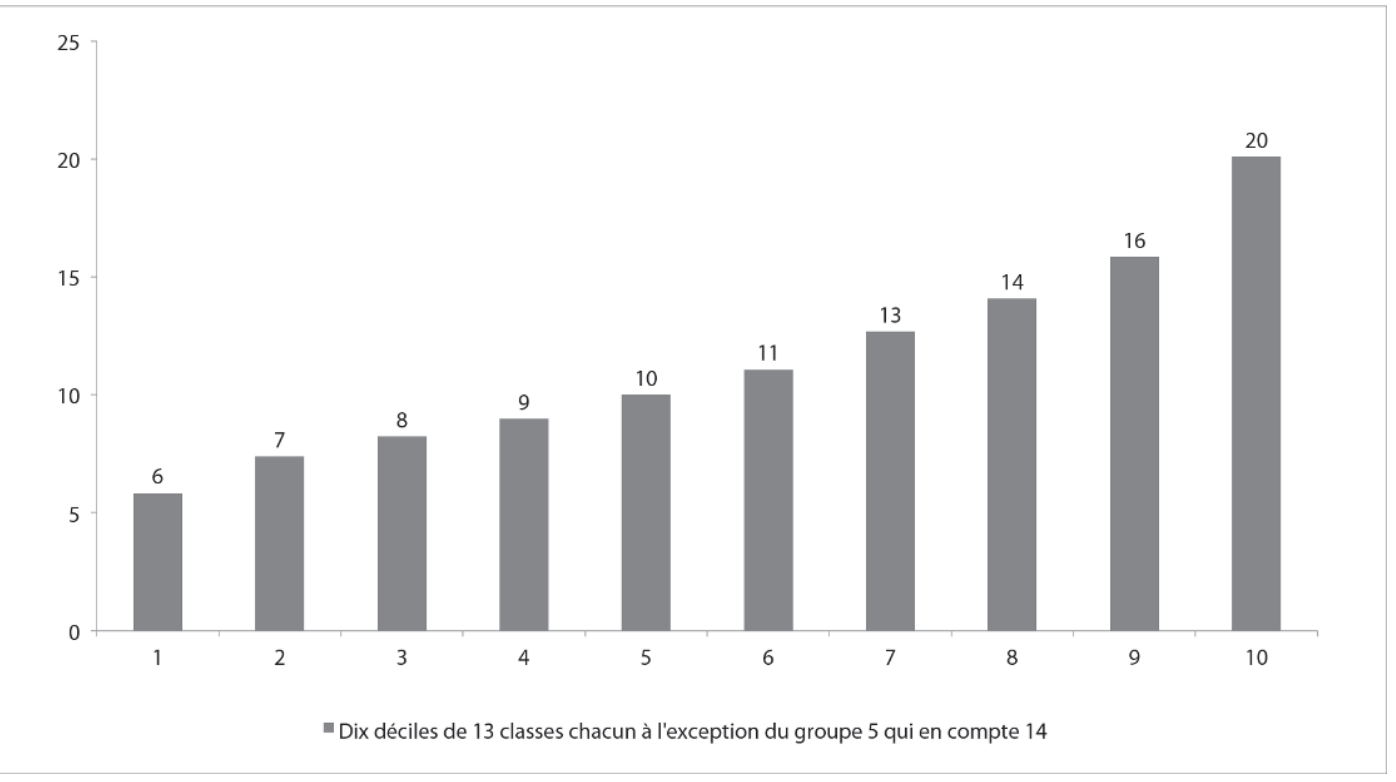

Note de lecture : le tempo moyen du premier décile est de 6, celui du dixième décile de 20.

Les relevés réalisés par les enquêteurs de la recherche Lire et Écrire pendant les neuf premières semaines de l'année scolaire nous ont permis d'établir la liste des correspondances graphophonémiques étudiées dans 131 classes et de les ranger par ordre décroissant de fréquence d'enseignement. Si l'on examine les 15 correspondances les plus fréquemment étudiées, on constate que 8 d'entre elles le sont par plus de la moitié des enseignants. II s'agit des

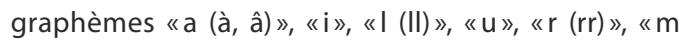
$(m m) », ~ « p(p p) »$, «o (ô)» et de leurs correspondants phonémiques respectifs (voir tableau 1).

Dans la progression pédagogique élaborée par Liliane Sprenger-Charolles (Dehaene, 2011), 24 graphèmes sont proposés avant la dixième semaine de classe. Sur ces 24 graphèmes, 11 sont communs avec ceux que les maîtres enseignent prioritairement. 7 des 8 correspondances enseignées par plus de la moitié des professeurs de notre enquête appartiennent à la programmation de Liliane Sprenger-Charolles, ce qui montre que les choix des enseignants sur le terrain sont cohérents avec ceux de notre collègue même s'ils s'en éloignent sur le plan du tempo.

Notons que l'étude de quelques graphèmes fréquents en français n'est pas souvent conduite explicitement dans les classes que nous avons observées.
5 d'entre eux, [n (nn), c (cc), d, i, e\#], n'apparaissent qu'entre le $19^{\mathrm{e}}$ et le $38^{\mathrm{e}}$ rang du classement que nous avons établi. Le «e » muet notamment possède la troisième fréquence la plus élevée en français, après celle des paires formées par les graphèmes «a » et « $r$ » et leurs correspondants phonémiques respectifs. Son étude est proposée en treizième position dans la programmation de Liliane Sprenger-Charolles mais elle apparaît seulement à la trente-huitième place dans notre classement.

\section{Résultats inférentiels}

Lorsque nous testons I'influence de la variable didactique tempo sur les performances des élèves en code, nous identifions une forme quadratique avec une valeur optimale de 15. Autrement dit, l'élévation du tempo influence significativement et positivement les apprentissages jusqu'à 15 correspondances graphophonémiques enseignées pendant les neuf premières semaines de classe $(p=0,052$; voir annexe 2 , modèle 4). Cet optimum est de 14 pour les élèves initialement faibles $(p=0,018)$. Pour ces derniers, nous avons également identifié un effet palier à $12(p=0,092)$, autrement dit une valeur de tempo à partir de laquelle les élèves obtiennent de meilleurs résultats par comparaison à ceux qui bénéficient de tempos moins 
Tableau 1. Correspondances graphophonémiques et graphèmes muets prioritairement enseignés par les maîtres de cours préparatoire ${ }^{a}$

\begin{tabular}{|c|c|c|c|c|c|c|}
\hline Rang & $\begin{array}{l}\text { Corres } \\
\text { graphop }\end{array}$ & $\begin{array}{l}\text { ondances } \\
\text { onémiques }\end{array}$ & $\begin{array}{l}\text { Pourcentages de } \\
\text { classes qui les }\end{array}$ & $\begin{array}{l}\text { Fréquences des } \\
\text { correspondances }\end{array}$ & $\begin{array}{l}\text { Ordre des } \\
\text { fréquences des }\end{array}$ & $\begin{array}{c}\text { Ordre d'étude } \\
\text { dans la }\end{array}$ \\
\hline 1 & a (à, â) & [a] ou [a] & 99,2 & 6,94 & 2 & 1 \\
\hline 2 & $\mathrm{i}$ & [i] & 91,6 & 6,53 & 4 & 3 \\
\hline 3 & I (II) & [l] & 81,7 & 4,52 & 7 & 6 \\
\hline 4 & u & [Y] & 77,9 & 1,72 & 17 & 5 \\
\hline 5 & $r(r r)$ & {$[R]$} & 77,1 & 7,86 & 1 & 7 \\
\hline 6 & $\mathrm{~m}(\mathrm{~mm})$ & [m] & 74,0 & 3,16 & 10 & $* c$ \\
\hline 7 & $p(p p)$ & [p] & 68,7 & 2,71 & 12 & 20 \\
\hline 8 & $\mathrm{o}(\hat{0})$ & [o] [0] & 64,1 & 5,07 & 6 & 4 \\
\hline 9 & e & [ə] [œ] [Ø] & 47,3 & 1,04 & 28 & 11 \\
\hline 10 & $\mathrm{t}(\mathrm{tt})$ & {$[\mathrm{t}]$} & 39,7 & 6,06 & 5 & 21 \\
\hline 11 & $y$ & [i] & 35,9 & 0,59 & 38 & * \\
\hline 12 & ou (où) & {$[u]$} & 32,8 & 0,76 & 35 & 10 \\
\hline 13 & $\mathrm{~s}(\mathrm{ss})$ & [s] & 29,8 & 3,27 & 9 & * \\
\hline 14 & é & [e] & 29,0 & 3,74 & 8 & 2 \\
\hline 15 & $\mathrm{au}$ & [o] [0] & 22,1 & 0,39 & 43 & * \\
\hline 19 & $\mathrm{n}(\mathrm{nn})$ & [n] & 16,8 & 2,89 & 11 & * \\
\hline 21 & $c(c c)$ & {$[\mathrm{k}]$} & 13,7 & 2,52 & 13 & * \\
\hline 22 & $d$ & [d] & 11,5 & 2,34 & 14 & 23 \\
\hline 27 & $\mathrm{i}$ & [j] (yod) & 6,9 & 1,92 & 15 & * \\
\hline 38 & e & $\#^{d}$ & 3,8 & 6,57 & 3 & 13 \\
\hline
\end{tabular}

Notes de lecture : a : afin de faciliter la lecture de notre tableau, nous avons choisi de présenter uniquement les lignes de correspondances graphèmes-phonèmes que nous commentons. C'est la raison pour laquelle le classement est incomplet; $b$ : nous avons établi les fréquences des correspondances graphèmes-phonèmes du français à partir des travaux de Ghneim (1997) qui, pour transcrire la chaîne écrite en une suite de caractères phonétiques, a analysé les 80000 entrées du dictionnaire Grand Robert; c : les cases sont marquées d'un astérisque (*) lorsque les correspondances graphèmes-phonèmes ne sont pas enseignées avant la dixième semaine de classe dans la progression théorique de Sprenger-Charolles; $d$ : \# signifie muet.

À noter que 5 correspondances parmi les 15 les plus fréquentes en français sont étudiées par moins de $17 \%$ des classes. Leur rang de classement selon leur fréquence d'étude dans l'échantillon est compris entre 19 et 38. 
Figure 2. Entrée privilégiée par les enseignants (valeurs exprimées en nombre de classes)

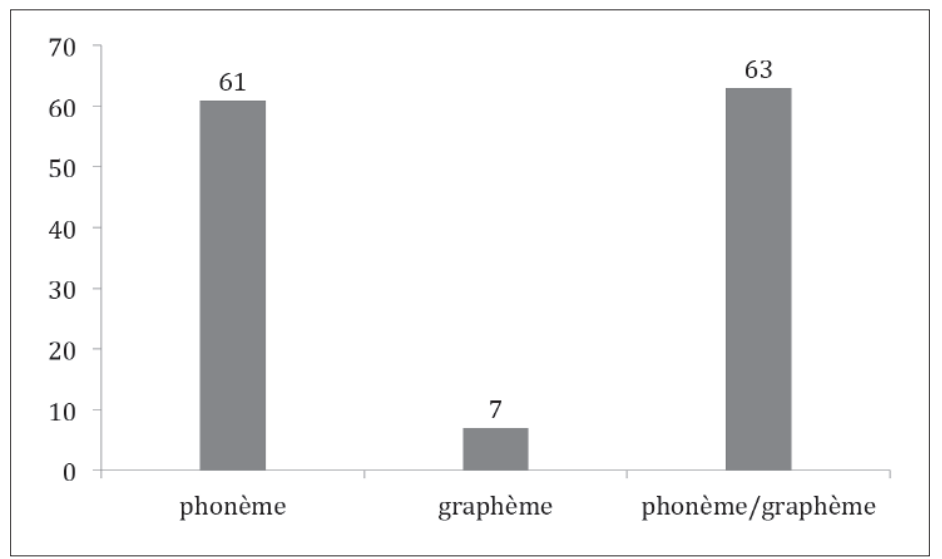

Figure 3. Rendement effectif des textes utilisés comme supports d'apprentissage (en \%)

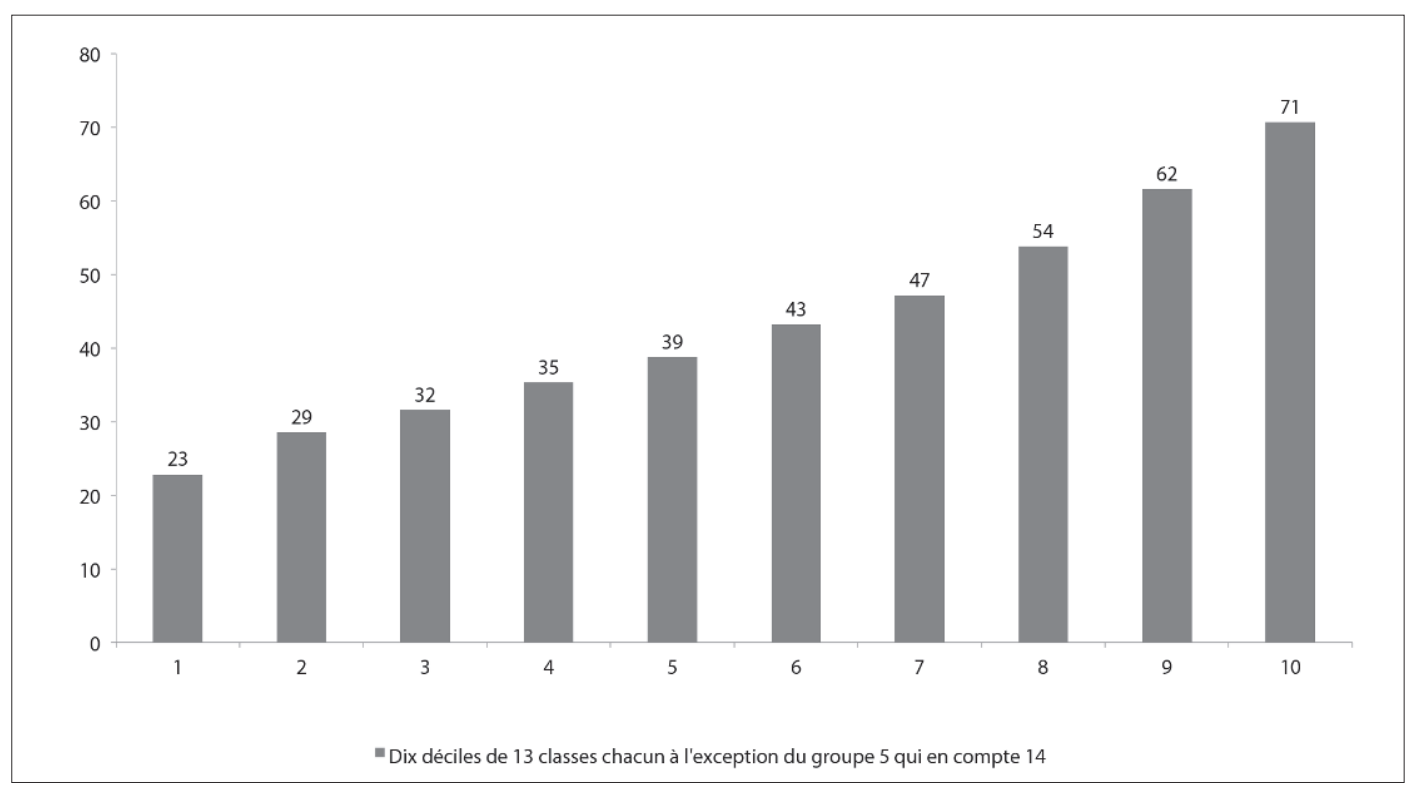

Note de lecture : le rendement effectif moyen du premier décile est de $23 \%$, celui du dixième décile de $71 \%$. 
rapides. Les élèves initialement faibles progressent donc davantage en décodage lorsque les tempos sont compris entre 12 et 14 . Pour les élèves initialement forts, plus le tempo est élevé, plus leurs progrès sont sensibles, et ceci sur l'ensemble de l'intervalle des valeurs observées, c'est-à-dire de 5 à $26(p=0,027)$.

\section{Variable didactique entreecgp}

\section{Résultats descriptifs}

Lorsqu'ils étudient une nouvelle correspondance graphophonémique, les enseignants observés dans la recherche Lire et Écrire privilégient l'entrée phonémique à l'entrée graphémique. Ils sont 61 à débuter leur enseignement par le phonème. Ils incitent leurs élèves à identifier, discriminer et localiser un nouveau phonème avant de présenter son ou ses correspondants graphémiques. 7 enseignants font le chemin inverse et commencent par le graphème. Mais dans presque la moitié des cas (48\%), les maîtres introduisent simultanément les deux, c'est-à-dire la correspondance graphophonémique, sans que l'on puisse distinguer l'antériorité de l'un par rapport à l'autre (voir figure 2).

\section{Résultats inférentiels}

Nous avons testé les effets de la variable didactique entreecgp en suivant les étapes que nous avons décrites dans le paragraphe "analyses multiniveaux». Nous n'avons identifié aucune influence de l'entrée choisie par les enseignants sur les performances des élèves en décodage.

\section{Variable didactique rendement effectif}

\section{Résultats descriptifs}

Dans notre étude, les rendements effectifs s'échelonnent de 11 à $76 \%$. Cela signifie que certains élèves de cours préparatoire peuvent déchiffrer seuls les trois quarts du texte là où d'autres peuvent en déchiffrer à peine plus d'un dixième. Entre ces deux extrêmes du paysage pédagogique français, les deux tiers des enseignants (66\%) proposent des textes dont les rendements effectifs sont compris entre 29 et $58 \%(-1 \sigma$ et $+1 \sigma)$. Le rendement effectif moyen de l'échantillon est de $43 \%$ ( $\sigma=14$ ) et la médiane se situe à $41 \%$ (voir figure 3 ).

\section{Résultats inférentiels}

Nous n'observons pas d'effet linéaire moyen de la mances en code des élèves pris dans leur ensemble. En revanche, nous observons une influence significative et positive du rendement effectif sur les performances des élèves qui obtiennent des scores intermédiaires en code $(p=0,031)$. Cet effet est optimal à $53 \%$, autrement dit les performances des élèves de niveau intermédiaire sont meilleures lorsque le rendement effectif tend vers cette valeur. Par ailleurs, la variable didactique catégorisée en trois modalités selon les bornes 29 et $57 \%$ permet de mettre en évidence des effets paliers. Les élèves qui apprennent à lire sur des textes déchiffrables à plus de $29 \%$ progressent davantage que les élèves qui apprennent à lire sur des textes moins déchiffrables $(p=0,074 ; p=0,015$; voir annexe 3 , modèle 5$)$. Cette influence est particulièrement nette pour les élèves de niveau intermédiaire $(p=0,028 ; p=$ $0,025)$, alors que pour les élèves initialement faibles, un effet significatif et positif est décelé pour des rendements effectifs supérieurs ou égaux à $57 \%(p=0,021)$. Dans tous les cas, les rendements effectifs inférieurs ou égaux à $29 \%$ pénalisent les apprentissages des élèves.

21 enseignants sur 131, soit $16 \%$, ont proposé à leurs élèves des supports d'apprentissage aux rendements effectifs inférieurs au palier que nous avons identifié. À l'opposé, les rendements effectifs moyens des deux derniers déciles sont supérieurs à $57 \%$ (voir figure 3 ) et 26 enseignants utilisent des supports d'apprentissage qui influencent significativement les performances des élèves initialement faibles en code.

\section{Discussion}

Les travaux anglo-saxons ont souligné l'intérêt d'un enseignement précoce et explicite des correspondances graphophonémiques (NRP, 2000). En revanche, ils n'ont pas relevé de différence significative d'efficacité entre les approches synthétiques et analytiques, autrement dit entre les démarches qui reposent sur l'association de lettres ou de groupes de lettres à des sons pour former des syllabes et des mots et celles qui consistent à nommer les syllabes et les mots pour ensuite identifier les correspondances lettres-sons qui les composent.

Nous souhaitions savoir si nous retrouverions ces résultats dans l'école française. Nous avons élaboré des modèles multiniveaux nous permettant de contrôler les performances initiales des élèves, leurs caractéristiques propres, les caractéristiques de la classe et celles des enseignants. Nos résultats vont dans le même sens que ceux de nos collègues anglo-saxons et confirment l'in- 
fluence significative d'une étude précoce et explicite du code alphabétique sur les performances des élèves en décodage. Ils ne révèlent pas non plus de différence entre les enseignants qui choisissent une entrée phonémique et ceux qui choisissent une entrée graphémique ou mixte : I'important est que les correspondances soient étudiées, quel que soit le sens de l'étude.

Lorsque nous avons testé les effets de la variable didactique tempo sur les performances des élèves en décodage, nous avons identifié 15 comme valeur optimale. Autrement dit, l'élévation du tempo influence significativement et positivement les performances des élèves jusqu'à un optimum de 15 correspondances graphophonémiques enseignées pendant les neuf premières semaines de classe. Cet optimum est de 14 pour les élèves qui obtiennent des scores faibles à l'entrée du cours préparatoire. Nous avons identifié un effet linéaire moyen qui s'étend sur l'ensemble des valeurs de l'étude, c'est-à-dire de 5 à 26, pour les élèves initialement forts en code. Nos analyses statistiques nous ont également permis de mettre en évidence des effets paliers, l'un à 11 en considérant l'ensemble des élèves de l'étude, l'autre à 12 en considérant seulement les élèves initialement faibles. Autrement dit, selon le niveau initial des élèves, les effets du tempo sont différents.

Le tempo moyen pratiqué par les 131 enseignants expérimentés de l'étude Lire et Écrire est très légèrement supérieur aux valeurs pénalisantes pour les apprentissages. Toutefois, 67 enseignants adoptent un tempo inférieur à 11 et pourraient être mis en alerte sur le bien-fondé de leurs choix, même s'il n'est pas exclu que d'autres caractéristiques de leur organisation didactique puissent compenser cette faiblesse.

95 enseignants suivent un tempo inférieur à 14, c'est-à-dire plus lent que la valeur optimale que nous avons identifiée pour les élèves initialement faibles, et 24 adoptent un tempo supérieur à 15 qui semble avant tout bénéficier aux élèves initialement forts. La très grande majorité des enseignants n'étudie donc pas suffisamment de correspondances graphophonémiques avant la dixième semaine de classe mais certains vont trop vite pour les élèves qui obtiennent des scores faibles à l'entrée du cours préparatoire. Le rythme d'enseignement le plus propice aux apprentissages des élèves initialement faibles équivaut à l'étude explicite de 3 correspondances graphophonémiques par quinzaine avant la dixième semaine de classe. Un enseignement plus rapide du code alphabétique, supérieur d'environ 3 correspondances à la moyenne de l'étude Lire et Écrire, améliorerait l'autonomie de déchiffrage des élèves et créerait des conditions plus favorables à leur apprentissage.

Les enseignants expérimentés de cours préparatoire étudient principalement des correspondances aux fréquences élevées mais jamais toutes ni exclusivement celles qui font partie des 15 plus fréquentes en français. Nous avons noté que seuls $77 \%$ des enseignants étudient la correspondance graphophonémique la plus fréquente ( $\ll r, r r » / R /$ ) avant la dixième semaine de classe, et qu'ils sont moins de $4 \%$ à étudier explicitement le «e» muet qui possède pourtant la troisième fréquence théorique la plus élevée. Si le "pouvoir déchiffrer» offert aux élèves est retenu comme facteur de réussite en lecture, des recommandations peuvent être faites sur la nature des correspondances à étudier en priorité, en particulier pour favoriser les apprentissages des élèves les plus dépendants de l'intervention pédagogique. Peut-être serait-il bon d'alerter les enseignants sur ce point, surtout si l'on fait I'hypothèse qu'ils n'en sont pas tous conscients.

Les comparaisons que nous avons effectuées plus haut entre les correspondances graphèmes-phonèmes prioritairement étudiées par les enseignants de cours préparatoire, les fréquences théoriques de ces correspondances en français et la proposition de Liliane Sprenger-Charolles, nous laissent penser qu'une progression de l'étude du code élaborée à partir du critère de fréquence est fondée. Toutefois, la planification de l'étude du code ne peut être pensée indépendamment du contenu linguistique des supports d'enseignement de la lecture : certains professeurs prennent garde à ce que les textes proposés aux élèves soient suffisamment déchiffrables et ils semblent avoir raison. En effet, nos résultats soulignent l'influence positive et significative du rendement effectif sur les performances des élèves en décodage, notamment lorsque ces derniers obtiennent des scores faibles ou intermédiaires en code à l'entrée au cours préparatoire. Ils confirment ainsi notre hypothèse de départ. Lorsque la part déchiffrable des textes est suffisante, elle accroît le sentiment de compétence et la clarté cognitive des élèves, favorise la mémorisation des correspondances graphophonémiques et génère un mécanisme d'auto-apprentissage, autrement dit elle induit davantage de progrès.

Les 26 classes qui présentent des rendements effectifs supérieurs à $57 \%$, autrement dit celles qui placent les élèves initialement faibles dans un contexte favorable pour développer leurs compétences en code, utilisent des supports d'enseignement issus de manuels de lecture qui relèvent d'approches extrêmement variées. En 
Tableau 2. Classes de l'étude Lire et Écrire qui présentent des rendements effectifs supérieurs à $57 \%$

\begin{tabular}{|c|c|c|c|}
\hline Identifiant classe & Manuel & Typologie des manuels & Rendements effectifs (en \%) \\
\hline 89 & Ribambelle (Hatier) & Approche intégrative & 57,08 \\
\hline 127 & Un monde à lire (Nathan) & Approche intégrative & 57,14 \\
\hline 103 & Taoki (Istra) & Approche syllabique & 59,09 \\
\hline 18 & Sans manuel & & 60,19 \\
\hline 1 & À tire-d'aile (Hatier) & Approche intégrative & 60,78 \\
\hline 6 & À l'école des albums (Retz) & Approche intégrative rapide & 61,43 \\
\hline 47 & Taoki (Istra) & Approche syllabique & 61,64 \\
\hline 79 & Bulle (Bordas) & Approche intégrative & 62,37 \\
\hline 54 & Léo et Léa (Belin) & Approche syllabique & 62,66 \\
\hline 119 & Léo et Léa (Belin) & Approche syllabique & 64,18 \\
\hline 5 & Sans manuel & & 64,72 \\
\hline 112 & Albums série libellule (SEDRAP) & Approche intégrative & 64,72 \\
\hline 108 & À l'école des albums (Retz) & Approche intégrative rapide & 64,77 \\
\hline 111 & Justine et compagnie (Belin) & Approche phonique & 64,97 \\
\hline 100 & À coup sûr (Istra) & Approche syllabique & 65,98 \\
\hline 105 & Un monde à lire (Nathan) & Approche intégrative & 66,4 \\
\hline 17 & À l'école des albums (Retz) & Approche intégrative rapide & 67,27 \\
\hline 78 & Lire avec Patati et Patata (Accès) & Approche intégrative & 67,68 \\
\hline 63 & Je lis, j'écris (Les lettres bleues) & Approche syllabique & 69,34 \\
\hline 113 & Léo et Léa (Belin) & Approche syllabique & 69,65 \\
\hline 68 & Sans manuel & & 72,83 \\
\hline 15 & Sans manuel & & 73,81 \\
\hline 80 & Léo et Léa (Belin) & Approche syllabique & 74,14 \\
\hline 61 & Je lis, j'écris (Les Lettres bleues) & Approche syllabique & 74,82 \\
\hline 131 & Je lis, j'écris (Les Lettres bleues) & Approche syllabique & 75,29 \\
\hline 91 & À coup sûr (Istra) & Approche syllabique & 76,29 \\
\hline
\end{tabular}

effet, 10 enseignants utilisent un manuel intégratif (Goigoux \& Cèbe, 2006), 11 un manuel syllabique ${ }^{8}$ et 1 s'appuie sur un manuel phonique 9 . Par ailleurs, 4 enseignants n'utilisent pas de manuel de lecture (voir tableau 2). En portant plus ou moins sciemment une attention particulière à l'autonomie de déchiffrage qu'ils offrent aux

8 Les manuels qui relèvent d'une approche syllabique sont basés sur un enseignement explicite des correspondances graphophonologiques (en partant des graphèmes) et excluent toute mémorisation de mots entiers. Ils proposent aux élèves des textes constitués exclusivement de graphèmes préalablement étudiés.

9 Les manuels qui relèvent d'une approche phonique privilégient l'entrée phonémique. Les textes proposés aux élèves sont écrits spécialement pour servir la progression de l'étude du code. élèves lors des leçons de lecture, les enseignants de ces classes créent les conditions de la réussite.

En 2000, les auteurs du National Reading Panel s'étonnaient que «très peu de recherches aient évalué la contribution de la part déchiffrable des textes à l'efficacité des programmes d'enseignement des correspondances graphèmes-phonèmes » (NRP, 2000, chapitre 2, p.97-98, traduit par nous). Depuis, seule une étude expérimentale portant sur des élèves susceptibles de rencontrer des difficultés de lecture a été menée (Vaughn, Denton \& Fletcher, 2010). Deux groupes d'élèves ont bénéficié d'un programme d'enseignement intensif de lecture de mots et de décodage à raison de 30 minutes par jour pendant 25 semaines, I'un a lu des textes fortement déchiffrables, l'autre des 
textes qui ne l'étaient pas, et un troisième groupe servant de contrôle n'a bénéficié d'aucun entraînement. Les résultats de cette étude soulignent l'influence significative et positive du programme d'enseignement intensif sur les apprentissages des élèves. En revanche, aucune conclusion n'a pu être tirée concernant les effets de la part déchiffrable des textes, notre hypothèse étant que ces effets ont été masqués par le programme d'entraînement intensif. Notre recherche peut donc être considérée comme pionnière sur le plan de l'évaluation de l'efficacité du rendement effectif sur les performances des élèves en décodage.

\section{Conclusion}

Dans cet article, nous faisons du tempo une variable didactique à part entière, indépendante de critères linguistiques, et nous montrons dans quelle mesure elle influence les performances des élèves en décodage, en particulier celles des élèves initialement faibles. Par ailleurs, en nous intéressant à la fréquence des correspondances graphophonémiques étudiées et $\mathrm{au}$ "pouvoir déchiffrer» qu'elles offrent aux élèves, nous tissons des liens entre le tempo et le contenu linguistique des textes servant de supports d'enseignement de la lecture. Le rendement effectif influence les performances des élèves en décodage, ce qui nous laisse penser qu'il serait utile de rendre cette variable didactique accessible aux enseignants ${ }^{10}$ afin qu'ils aient

10 Nous avons conçu un outil d'analyse automatique nommé Anagraph qui permet de mesurer la part déchiffrable des textes qui servent de supports d'enseignement de la lecture. En ligne: $<$ http:// anagraph.ens-lyon.fr/web/app.php> (consulté le 17 octobre 2017).

\section{Bibliographie}

ATTALI A. \& BRESSOUX P. (2002). L'évaluation des pratiques éducatives dans les premier et second degrés. Rapport pour le Haut Conseil de l'Évaluation de l'École (HCEE). Paris : HCEE.

BRAIBANT J.-M. \& GÉRARD F.-M. (1996). «Savoir lire : une question de méthodes?» Bulletin de psychologie scolaire et d'orientation, $\mathrm{n}^{\circ} 1, \mathrm{p} .7-45$.

BRESSOUX P. (2010). Méthodes en sciences humaines. Modélisation statistique appliquée aux sciences sociales. Bruxelles : De Boeck, $2^{\mathrm{e}}$ éd.

BRESSOUX P. (2011). «Effet-maître et pratiques de classe». In E. Bourgeois \& G. Chapelle (dir.), Apprendre et faire apprendre. Paris : PUF, $2^{\mathrm{e}}$ éd., p. 221-231.

CATACH N. (2012). L'orthographe française, l'orthographe en connaissance de la part déchiffrable des textes qu'ils utilisent lors des leçons de lecture.

Nos travaux de recherche avaient pour objectif de produire des connaissances sur les caractéristiques des pratiques d'enseignement efficaces et équitables afin de nourrir la réflexion sur le pilotage du système éducatif. Ils avaient aussi pour ambition d'aider les maîtres à circonscrire les choix qui s'offrent à eux et d'éclairer leurs propres pratiques. En donnant aux enseignants des repères temporels facilitant la planification de l'étude du code alphabétique et en faisant du rendement effectif un indicateur de réussite des apprentissages, nous espérons que nos résultats serviront à la conception et à la conduite des enseignements dans les classes de cours préparatoire. Ces résultats ont été obtenus en observant le monde pédagogique tel qu'il est, sans transformation des pratiques de classe. Ainsi, leur caractère écologique devrait en faciliter I'appropriation.

Jérôme Riou

Université Clermont-Auvergne, ACTé (EA 4281), École normale supérieure de Lyon, Institut français de l'éducation jerome.riou@ens-lyon.fr

Valérie Fontanieu École normale supérieure de Lyon, Institut français de l'éducation valerie.fontanieu@ens-lyon.fr

leçons: un traité théorique et pratique. Paris : Armand Colin, $3^{\mathrm{e}}$ éd.

CHAUVEAU G. \& ROGOVAS-CHAUVEAU E. (1989). «Les idées des enfants de six ans sur la lecture et l'écriture». Psychologie scolaire, $\mathrm{n}^{\circ} 68, \mathrm{p} .7-28$.

CUSSET P.-Y. (2014). «Les pratiques pédagogiques efficaces. Conclusions de recherches récentes». Document de travail no2014-01, France Stratégie. En ligne : <http://www.strategie.gouv.fr/sites/strategie.gouv.fr/files/atoms/files/doc_ de_travail_12aout_vupyc-11-09.pdf > (consulté le 8 septembre 2017).

DEHAENE S. (dir.) (2011). Apprendre à lire. Des sciences cognitives à la salle de classe. Paris : Odile Jacob. 
DOWNING J. \& FIJALKOW J. (1984). Lire et raisonner. Toulouse : Privat.

DURU-BELLAT M. \& MINGAT A. (1998). «Le déroulement de la scolarité au collège : le contexte fait des différences». Revue française de sociologie, $\mathrm{n}^{\circ} 29$, p. 649-666.

ECALLE J. \& MAGNAN A. (2015). L'apprentissage de la lecture et ses difficultés. Paris : Dunod, $2^{\mathrm{e}}$ éd.

FEYFANT A. \& GAUSSEL M. (2007). « Méthodes de lecture et difficultés d'apprentissage». Dossier d'actualité Veille et Analyse, $\mathrm{n}^{\circ} 31$. En ligne : <http://ife.ens-lyon.fr/vst/LettreVST/pdf/31-novembre-2007.pdf> (consulté le 8 septembre 2017).

GENTAZ, SPRENGER-CHAROLLES L., COLÉ P. \& LE CAM M. (2013). «Évaluation quantitative d'un entraînement à la lecture à grande échelle pour des enfants de CP scolarisés en réseaux d'éducation prioritaire : apports et limites». $A N A E ́, \mathrm{n}^{\circ} 123$, p. $172-181$.

GENTAZ E., SPRENGER-CHAROLLES L. \& THEUREL A. (2015). «Differences in the Predictors of Reading Comprehension in First Graders from Low Socio-Economic Status Families with Either Good or Poor Decoding Skills». PLoS ONE, 10(3) : e0119581. En ligne : <https://doi.org/10.1371/journal. pone.0119581> (consulté le 8 septembre 2017).

GHNEIM N. (1997). Relations entre les codes de l'oral et de l'écrit: contraintes et ambiguittés. Thèse de doctorat non publiée, sciences du langage, université Stendhal-Grenoble 3.

GOIGOUX R. (1993a). L'apprentissage initial de la lecture. De la didactique à la psychologie cognitive (étude longitudinale). Thèse de doctorat, sciences de l'éducation, université René-Descartes-Paris 5, Sorbonne Sciences humaines.

GOIGOUX R. (1993b). "L'enseignement de la lecture au cycle 2 en débat». Les actes de lecture, n 43, p.56-63. En ligne : <http://www.lecture.org/revues_livres/actes_lectures/AL/AL43/AL43P56.pdf> (consulté le 8 septembre 2017).

GOIGOUX R. (2000). «Apprendre à lire à l'école : les limites d'une approche idéovisuelle». Psychologie française, vol. $45, n^{\circ} 3$, p. 235-245.

GOIGOUX R. \& CÈBE S. (2006). Apprendre à lire à l'école. Tout ce qu'il faut savoir pour accompagner l'enfant. Paris : Retz.

GOIGOUX R., JARLÉGAN A. \& PIQUÉE C. (2015). «Évaluer l'influence des pratiques d'enseignement du lire-écrire sur les apprentissages des élèves : enjeux et choix méthodologiques ». Recherches en didactique, $\mathrm{n}^{\circ} 17$, p. 33-52.

GOMBERT J.-É., COLÉ P., VALDOIS S., GOIGOUX R., MOUSTY P. \& FAYOL M. (2000). Enseigner la lecture. Apprendre à lire au cycle 2. Paris : Nathan.

INSTITUT NATIONAL DE LA SANTE ET DE LA RECHERCHE MEDICALE [INSERM] (2007). Dyslexie, dysorthographie, dyscalculie : bilan des données scientifiques. Paris : Inserm. En ligne : <http://ipubli-inserm.inist.fr/handle/10608/73> (consulté le 8 septembre 2017).

LÉTÉ B., SPRENGER-CHAROLLES L. \& COLÉ P. (2004). "Manulex: A grade-level lexical database from French elementary-school readers». Behavior Research Methods, Instruments, and Computers, vol.36, n¹, p.156-166.
MINISTÈRE DE L'ÉDUCATION NATIONALE (2002). «Horaires et programmes de l'enseignement de l'école primaire». Bulletin officiel de l'Éducation nationale, 14 février, $\mathrm{n}^{\circ} 1$, p. 44-45.

NATIONAL READING PANEL (2000). Teaching children to read: An Evidence-Based Assessment of the Scientific Research Literature on Reading and Its Implications for Reading Instruction. Bethsda (États-Unis) : National Institute of Child Health and Human Development (NICHD).

NONNON É. \& GOIGOUX R. (2007). «Travail de l'enseignant, travail de l'élève dans l'apprentissage de la lecture au cycle II ». Repères, n³6, p.5-36.

ORTÉGA É. \& LÉTÉ B. (2010). eManulex : Electronic version of Manulex and Manulex-infra databases. En ligne : <http:// www.manulex.org> (consulté le 8 septembre 2017).

PEEREMAN R., LÉTÉ B. \& SPRENGER-CHAROLLES L. (2007). «Manulex-infra: Distributional characteristics of grapheme-phoneme mappings, infra-lexical and lexical units in child-directed written material». Behavior Research Methods, vol.39, n³, p.593-603.

PROGRAMME INCITATIF DE RECHERCHE EN ÉDUCATION ET FORMATION [PIREF] (2003). L'enseignement de la lecture à l'école primaire. Des premiers apprentissages au lecteur compétent. Conférence de consensus organisée à Paris les 4 et 5 décembre 2003. En ligne: $<$ http://www.cndp.fr/bienlire/01-actualite/c-en-parle06.asp > (consulté le 8 septembre 2017).

SAUSSEZ F. \& LESSARD C. (2009). «Entre orthodoxie et pluralisme, les enjeux de l'éducation basée sur la preuve». Revue française de pédagogie, $\mathrm{n}^{\circ} 168, \mathrm{p} .111-136$. En ligne : <http://rfp.revues.org/1804> (consulté le 8 septembre 2017).

SHARE D. L. (1995). «Phonological recoding and self-teaching: Sine qua non of reading acquisition». Cognition, $\mathrm{n}^{\circ} 55, \mathrm{p} .151-218$

SPRENGER-CHAROLLES L. \& COLÉ P. (2006). Pratiques pédagogiques et apprentissage de la lecture. CRAP. Cahiers pédagogiques.

SPRENGER-CHAROLLES, L., COLÉ P., BÉCHENNEC, D. \& KIPFFER-PIQUARD A. (2005). «French normative data on reading and related skills: From 7 to 10 year-olds». European Review of Applied Psychology/Revue européenne de Psychologie appliquée, n55, p.157-186.

VAN DAMME J., OPDEAKKER M.-C., VAN LANDEGHEM G. et al. (2009). «Fondements et principaux résultats de recherche sur l'efficacité dans l'enseignement». In X. Dumay \& V. Dupriez (dir.), L'efficacité dans l'enseignement. Bruxelles : De Boeck, p.17-34.

VAUGHN S., DENTON C. A. \& FLETCHER J. M. (2010). «Why intensive interventions are necessary for students with severe reading difficulties». Psychology in the Schools, vol.47, n5, p.432-444. En ligne: <http://dx.doi.org/10.1002/ pits.20481> (consulté le 8 septembre 2017). 
Annexe 1. Tableau de relevé d'étude du code

\begin{tabular}{|c|c|c|c|c|c|c|c|c|}
\hline $\mathrm{N}^{\circ}$ & $\begin{array}{l}\text { Correspo } \\
\text { graphèm }\end{array}$ & $\begin{array}{l}\text { ances } \\
\text { phonèmes }\end{array}$ & Exemples & $\begin{array}{c}\text { Date } 1^{\text {re }} \\
\text { trace }\end{array}$ & $\begin{array}{c}\text { Date } \\
\text { Institu. }\end{array}$ & $\begin{array}{c}\text { Aide- } \\
\text { mémoire }\end{array}$ & P, G ou PG & Remarques \\
\hline 1 & $a(\grave{a}, \hat{a})$ & [a] ou [a] & avocat, patte, pâte & & & & & \\
\hline 2 & $a i$ & {$[\varepsilon]$ ou $[e]$} & aimer, serai & & & & & \\
\hline 3 & ain (aim) & {$[\tilde{\varepsilon}]$} & pain, daim & & & & & \\
\hline 4 & an (am) & [ã] & enfant, ampoule & & & & & \\
\hline 5 & $a u$ & [о] [०] & autant, autobus & & & & & \\
\hline 6 & $b$ & [b] & bleu & & & & & \\
\hline 7 & $c(c c)$ & {$[\mathrm{k}]$} & couleur, accomplir & & & & & \\
\hline 8 & $c(c ̧)$. & {$[\mathrm{s}]$} & cerise, déçu & & & & & \\
\hline 9 & $c h$ & []] & chat & & & & & \\
\hline 10 & $d$ & [d] & deux & & & & & \\
\hline 11 & $e$ & [ə] [oe] [Ø] & devenir, lime & & & & & \\
\hline 12 & $e$ & [e] & dessin, effort & & & & & \\
\hline 13 & $e$ & {$[\varepsilon]$} & vert, mer & & & & & \\
\hline 14 & é & [e] & école, été & & & & & \\
\hline 15 & $\grave{e}(\hat{e})$ & {$[\varepsilon]$} & espèce, rêve & & & & & \\
\hline 16 & eau & [o] & bureau & & & & & \\
\hline 17 & ei (ey) & [e] ou $[\varepsilon]$ & reine, hockey & & & & & \\
\hline 18 & ein (eim) & {$[\tilde{\varepsilon}]$} & rein, Reims & & & & & \\
\hline 19 & en & {$[\tilde{\varepsilon}]$} & rien & & & & & \\
\hline 20 & en (em) & [ã] & enfant, emporter & & & & & \\
\hline 21 & er (et) & {$[e]$ ou $[\varepsilon]$} & manger, jouet & & & & & \\
\hline 22 & es (ez) & [e] ou $[\varepsilon]$ & tu es, nez & & & & & \\
\hline 23 & eu & [oe] ou [Ø] & peur, bleu & & & & & \\
\hline 24 & $e u$ & {$[\mathrm{Y}]$} & j'ai eu & & & & & \\
\hline 25 & $f(f f)$ & [f] & fou, effort & & & & & \\
\hline 26 & $g(e)$ & [3] & plage, plongeon & & & & & \\
\hline 27 & $g(g u)$ & [g] & goutte, guépard & & & & & \\
\hline 28 & $g n$ & $n$ & mignon & & & & & \\
\hline 29 & $i$ & [i] & ami & & & & & \\
\hline 30 & $i$ & [j] (yod) & avion & & & & & \\
\hline 31 & ill (il) & [j] (yod) & œil, famille & & & & & \\
\hline 32 & in (im) & {$[\tilde{\varepsilon}]$} & sapin & & & & & \\
\hline 33 & $j$ & [3] & jaune & & & & & \\
\hline 34 & $k$ & {$[\mathrm{k}]$} & kilo & & & & & \\
\hline 35 & $I(I I)$ & {$[1]$} & stylo, bulle & & & & & \\
\hline 36 & $m(m m)$ & {$[\mathrm{m}]$} & mon, femme & & & & & \\
\hline 37 & $n(n n)$ & [n] & niche, bonne & & & & & \\
\hline 38 & $O(\hat{o})$ & [о] [0] & stylo, école & & & & & \\
\hline 39 & oeu (oe) & [oe] ou [Ø] & cœur, vœu, œil & & & & & \\
\hline 40 & 00 & [o] [u] & zoo, foot & & & & & \\
\hline
\end{tabular}




\begin{tabular}{|c|c|c|c|c|c|c|c|c|}
\hline \multirow{2}{*}{$\begin{array}{l}N^{\circ} \\
41 \\
\end{array}$} & \multicolumn{2}{|c|}{$\begin{array}{l}\text { Correspondances } \\
\text { graphèmes/phonèmes }\end{array}$} & \multirow{2}{*}{$\begin{array}{l}\text { Exemples } \\
\text { roi } \\
\end{array}$} & \multirow[t]{2}{*}{$\begin{array}{c}\text { Date } 1^{\text {re }} \\
\text { trace }\end{array}$} & \multirow[t]{2}{*}{$\begin{array}{c}\text { Date } \\
\text { Institu. }\end{array}$} & \multirow[t]{2}{*}{$\begin{array}{c}\text { Aide- } \\
\text { mémoire }\end{array}$} & \multirow[t]{2}{*}{ P, G ou PG } & \multirow[t]{2}{*}{ Remarques } \\
\hline & $o i$ & [wa] & & & & & & \\
\hline 42 & oin & {$[\mathrm{w} \tilde{\varepsilon}]$} & coin & & & & & \\
\hline 43 & on (om) & [õ] & salon, pompe & & & & & \\
\hline 44 & ou (où) & {$[\mathrm{u}]$} & fou, où & & & & & \\
\hline 45 & $p(p p)$ & [p] & père, apporte & & & & & \\
\hline 46 & $p h$ & {$[f]$} & phoque & & & & & \\
\hline 47 & $q(q u)$ & {$[\mathrm{k}]$} & coq, quatre & & & & & \\
\hline 48 & $r(r r)$ & {$[\mathrm{R}]$} & rouge, arrêt & & & & & \\
\hline 49 & $s(s s)$ & [s] & soir, assis & & & & & \\
\hline 50 & $s$ & {$[z]$} & usé & & & & & \\
\hline 51 & $t(t t)$ & {$[\mathrm{t}]$} & toupie, belette & & & & & \\
\hline 52 & $t$ & {$[s]$} & solution, patient & & & & & \\
\hline 53 & $u$ & {$[\mathrm{Y}]$} & usine & & & & & \\
\hline 54 & $u$ & [0] & album & & & & & \\
\hline 55 & $u(i)$ & [ui] & pluie & & & & & \\
\hline 56 & un & 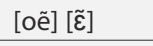 & brun & & & & & \\
\hline 57 & $v$ & {$[\mathrm{v}]$} & voyage & & & & & \\
\hline 58 & $w$ & {$[w]$} & web & & & & & \\
\hline 59 & $w$ & {$[v]$} & wagon & & & & & \\
\hline 60 & $x$ & [gz] ou [ks] & examen, axe & & & & & \\
\hline 61 & $y$ & [i] & analyse & & & & & \\
\hline 62 & $y$ & [j] (yod) & voyage & & & & & \\
\hline 63 & $z$ & {$[\mathrm{z}]$} & zoo & & & & & \\
\hline
\end{tabular}

\begin{tabular}{|c|c|c|c|c|c|c|c|c|}
\hline \multicolumn{9}{|c|}{ Lettres muettes : \# } \\
\hline $\mathrm{N}^{\circ}$ & Graphèmes & & Exemples & $\begin{array}{l}\text { Date } 1^{\text {re }} \\
\text { trace }\end{array}$ & $\begin{array}{l}\text { Date } \\
\text { Institu. }\end{array}$ & $\begin{array}{l}\text { Aide- } \\
\text { mémoire }\end{array}$ & $P, G$ ou $P G$ & Remarques \\
\hline 64 & c & $\#$ & blanc & & & & G & \\
\hline 65 & $d$ & $\#$ & rond & & & & G & \\
\hline 66 & $e$ & $\#$ & fillette & & & & G & \\
\hline 67 & $f$ & $\#$ & clef & & & & G & \\
\hline 68 & $g$ & $\#$ & sang & & & & G & \\
\hline 69 & $h$ & $\#$ & habit & & & & G & \\
\hline 70 & 1 & $\#$ & fils & & & & G & \\
\hline 71 & $p$ & $\#$ & loup & & & & G & \\
\hline 72 & $s$ & $\#$ & tapis & & & & G & \\
\hline 73 & $t$ & $\#$ & soldat & & & & G & \\
\hline 74 & $x$ & $\#$ & deux & & & & G & \\
\hline 75 & $z$ & $\#$ & riz & & & & G & \\
\hline
\end{tabular}


Annexe 2. Modèles multiniveaux estimant l'influence des variables didactiques tempo et tempo*tempo sur les performances finales des élèves en décodage

\begin{tabular}{|c|c|c|c|c|}
\hline Paramètres & $\begin{array}{l}\text { Modèle } 0 \\
\text { Vide } \\
2507 \text { élèves }\end{array}$ & $\begin{array}{l}\text { Modèle } 1 \\
\text { Performance } \\
\text { initiale en code } \\
2507 \text { élèves }\end{array}$ & $\begin{array}{l}\text { Modèle } 3 \\
\text { Variables de } \\
\text { contrôle de } \\
\text { niveaux } 1 \text { et } 2 \\
2507 \text { élèves }\end{array}$ & $\begin{array}{l}\text { Modèle } 4 \\
\text { Variables } \\
\text { didactiques tempo } \\
\text { et tempo*tempo } \\
2507 \text { élèves }\end{array}$ \\
\hline $\begin{array}{l}\text { Effets fixes } \\
\text { Constante }\end{array}$ & $-0,004(0,035)$ & $0,002(0,028)$ & $0,072(0,147)$ & $0,428(0,258)$ \\
\hline $\begin{array}{l}\text { Variables de niveau } 1 \\
\text { Score individuel initial (codez) } \\
\text { Sexe de l'élève (fille) } \\
\text { PCS défavorisé } \\
\text { PCS intermédiaire } \\
\text { Élève ayant redoublé } \\
\text { Élève né entre mai et août } \\
\text { Élève né à partir de septembre } \\
\text { Élève parlant une autre langue que le } \\
\text { français à la maison } \\
\text { Élève parlant le français et une autre } \\
\text { langue à la maison }\end{array}$ & & $0,622(0,015)^{* * * *}$ & $\begin{array}{l}0,590(0,016)^{* * *} \\
-0,023(0,029) \\
-0,173(0,045)^{* * *} \\
-0,063(0,045) \\
-0,664(0,077)^{* * *} \\
-0,022(0,037) \\
-0,019(0,038) \\
-0,163(0,064)^{*} \\
0,045(0,041)\end{array}$ & $\begin{array}{l}0,590(0,016)^{* * *} \\
-0,023(0,029) \\
-0,173(0,045)^{* * *} \\
-0,064(0,045) \\
-0,667(0,077)^{* * *} \\
-0,021(0,037) \\
-0,018(0,038) \\
-0,158(0,064)^{* *} \\
0,051(0,041)\end{array}$ \\
\hline $\begin{array}{l}\text { Variables de niveau } 2 \\
\text { Rythme scolaire (4,5 j/semaine) } \\
\text { Éducation prioritaire } \\
\text { Niveau moyen initial dans l'épreuve } \\
\text { Hétérogénéité initiale dans l'épreuve } \\
\% \text { PCS favorisé } \\
\text { Ancienneté au cours préparatoire }\end{array}$ & & & $\begin{array}{r}-0,114(0,070) \\
-0,003(0,071) \\
-0,065(0,087) \\
0,058(0,130) \\
0,154(0,179) \\
0,004(0,005)\end{array}$ & $\begin{array}{c}-0,121(0,069)^{*} \\
0,029(0,072) \\
-0,070(0,086) \\
0,095(0,129) \\
0,247(0,181) \\
0,004(0,005)\end{array}$ \\
\hline $\begin{array}{l}\text { Effet de la variable tempo } \\
\text { tempo } \\
\text { tempo*tempo (forme quadratique) }\end{array}$ & & & & $\begin{array}{l}0,065757(0,030)^{* *} \\
-0,002121(0,001)^{*}\end{array}$ \\
\hline $\begin{array}{l}\text { Effets aléatoires } \\
\text { Niveau } 2 \\
\text { Variance inter-classes } \\
\text { Niveau } 1 \\
\text { Variance intra-classe }\end{array}$ & $\begin{array}{l}0,111(0,020) \\
0,887(0,026)\end{array}$ & $\begin{array}{l}0,074(0,013) \\
0,535(0,016)\end{array}$ & $\begin{array}{l}0,068(0,012) \\
0,509(0,015)\end{array}$ & $\begin{array}{l}0,064(0,011) \\
0,509(0,015)\end{array}$ \\
\hline Déviance $(-2 \log \mathrm{L})$ & 6973,05 & 5714,06 & 5529,33 & 5523,91 \\
\hline
\end{tabular}

Notes de lecture : seuils de significativité : ${ }^{*}: p<0,10 ;{ }^{* *}: p<0,05 ;^{* * *}: p<0,01$. Les erreurs-types des coefficients figurent entre parenthèses.

Effet quadratique : l'extremum de la forme quadratique est de 15,5.

Significativité des modèles :

- le modèle 1 estime 1 paramètre supplémentaire par rapport au modèle vide. La décroissance de la déviance suit donc une loi du Chi2 à 1 degré de liberté : $\Delta(0-1)=6973,05-5714,06=1258,99(p<0,001)$;

- le modèle 2 (variables de contrôle de niveau 1) estime 8 paramètres supplémentaires par rapport au modèle 1. La décroissance de la déviance suit donc une loi du Chi2 à 8 degrés de liberté : $\Delta(1-2)=5714,06-5534,95=179,11$ $(\mathrm{p}<0,001)$;

- le modèle 3 estime 6 paramètres supplémentaires par rapport au modèle 2. La décroissance de la déviance suit donc une loi du Chi2 à 6 degrés de liberté : $\Delta(2-3)=5534,95$ - 5529,33 = 5,62 (modèle non significatif);

- le modèle 4 estime 2 paramètres supplémentaires par rapport au modèle 3 . La décroissance de la déviance suit une loi du Chi2 à 2 degrés de liberté : $\Delta(3-5)=5529,33-5523,91=5,42(p=0,067)$.

Variable d'interaction : la variable d'interaction entre les performances initiales des élèves en code et le tempo n'est pas significative. 
Annexe 3. Modèles multiniveaux estimant l'influence de la variable à trois modalités rend_eff_29_57 sur les performances finales en décodage des élèves initialement faibles en code

\begin{tabular}{|c|c|c|c|c|}
\hline Paramètres & $\begin{array}{l}\text { Modèle } 0 \\
(-0,5 \sigma) \\
\text { Vide } \\
706 \text { élèves }\end{array}$ & $\begin{array}{l}\text { Modèle } 1 \\
(-0,5 \sigma) \\
\text { Performance } \\
\text { initiale en } \\
\text { code706 élèves }\end{array}$ & $\begin{array}{l}\text { Modèle } 3 \\
(-0,5 \sigma) \\
\text { Variables de } \\
\text { contrôle de } \\
\text { niveau } 1 \text { et } 2 \\
706 \text { élèves }\end{array}$ & $\begin{array}{l}\text { Modèle } 5 \\
\quad(-0,5 \sigma) \\
\text { Variables à trois } \\
\text { modalités rend_ } \\
\text { eff_29_57 } \\
706 \text { élèves }\end{array}$ \\
\hline $\begin{array}{l}\text { Effets fixes } \\
\text { Constante }\end{array}$ & $-0,766(0,050)^{* * *}$ & $-0,075(0,085)$ & $0,063(0,282)$ & $-0,124(0,300)$ \\
\hline $\begin{array}{l}\text { Variables de niveau } 1 \\
\text { Score individuel initial (codez) } \\
\text { Sexe de l'élève (fille) } \\
\text { PCS défavorisé } \\
\text { PCS intermédiaire } \\
\text { Élève ayant redoublé } \\
\text { Élève né entre mai et août } \\
\text { Élève né à partir de septembre } \\
\text { Élève parlant une autre langue que le } \\
\text { français à la maison } \\
\text { Élève parlant le français et une autre } \\
\text { langue à la maison }\end{array}$ & & $0,661(0,067)^{* * *}$ & $\begin{array}{l}0,635(0,070)^{* * *} \\
-0,025(0,068) \\
-0,453(0,120)^{* * *} \\
-0,265(0,128)^{* *} \\
-0,375(0,152)^{* *} \\
-0,002(0,093) \\
0,069(0,092) \\
-0,160(0,109) \\
0,034(0,094)\end{array}$ & $\begin{array}{l}0,627(0,070)^{* * *} \\
-0,022(0,068) \\
-0,462(0,120)^{* * *} \\
-0,285(0,128)^{* *} \\
-0,372(0,152)^{* *} \\
0,000(0,093) \\
0,067(0,092) \\
-0,168(0,109) \\
0,056(0,094)\end{array}$ \\
\hline $\begin{array}{l}\text { Variables de niveau } 2 \\
\text { Rythme scolaire }(4,5 \mathrm{j} / \text { semaine }) \\
\text { Éducation prioritaire } \\
\text { Niveau moyen initial dans l'épreuve } \\
\text { Hétérogénéité initiale dans l'épreuve } \\
\% \text { PCS favorisé } \\
\text { Ancienneté au cours préparatoire }\end{array}$ & & & $\begin{array}{r}-0,225(0,123)^{*} \\
0,064(0,122) \\
-0,065(0,153) \\
0,204(0,223) \\
-0,243(0,348) \\
0,010(0,009) \\
\end{array}$ & $\begin{array}{l}-0,252(0,122)^{* *} \\
0,083(0,121) \\
-0,036(0,149) \\
0,212(0,217) \\
-0,229(0,342) \\
0,009(0,009)\end{array}$ \\
\hline $\begin{array}{l}\text { Effet de la variable rend_eff_29_57 } \\
\text { rend_eff_29_57 (1) } \\
\text { (référence: valeurs } \leq \text { à 29\%) } \\
\text { rend_eff_29_57 (2) } \\
\text { rend_eff_29_57 (3) }\end{array}$ & & & & $\begin{array}{l}0,177(0,135) \\
0,372(0,159)^{* *}\end{array}$ \\
\hline $\begin{array}{l}\text { Effets aléatoires } \\
\text { Niveau } 2 \\
\text { Variance inter-classes } \\
\text { Niveau } 1 \\
\text { Variance intra-classe }\end{array}$ & $\begin{array}{l}0,135(0,039) \\
0,895(0,052)\end{array}$ & $\begin{array}{l}0,145(0,037) \\
0,773(0,045)\end{array}$ & $\begin{array}{l}0,126(0,035) \\
0,741(0,044)\end{array}$ & $\begin{array}{l}0,113(0,032) \\
0,742(0,044)\end{array}$ \\
\hline Déviance (- $2 \log \mathrm{L})$ & 1998,895 & 1908,629 & 1841,418 & 1835,944 \\
\hline
\end{tabular}

Notes de lecture : seuils de significativité $:{ }^{*}: p<0,10 ;{ }^{* *}: p<0,05{ }^{* * *}: p<0,01$. Les erreurs-types des coefficients figurent entre parenthèses.

Significativité des modèles :

- le modèle $1(-0,5 \sigma)$ estime 1 paramètre supplémentaire par rapport au modèle vide $(-0,5 \sigma)$. La décroissance de la déviance suit donc une loi du Chi2 à 1 degré de liberté : $\Delta(0-1)=1998,90-1908,63=90,27(p<0,01)$.

- le modèle $2(-0,5 \sigma)$ (variables de contrôle de niveau 1) estime 8 paramètres supplémentaires par rapport au modèle $1(-0,5 \sigma)$. La décroissance de la déviance suit donc une loi du Chi2 à 8 degrés de liberté $: \Delta(1-2)=1908,63-1849,56=$ $59,07(p<0,01)$.

- le modèle $3(-0,5 \sigma)$ estime 6 paramètres supplémentaires par rapport au modèle $2(-0,5 \sigma)$. La décroissance de la déviance suit donc une loi du Chi2 à 6 degrés de liberté : $\Delta(2-3)=1849,56$ - 1841,42 = 8,14 (modèle non significatif).

- le modèle $5(-0,5 \sigma)$ estime 2 paramètres supplémentaires par rapport au modèle $3(-0,5 \sigma)$. La décroissance de la déviance suit une loi du Chi2 à 2 degrés de liberté : $\Delta(3-10)=1841,418-1835,944=5,474(p<0.10)$.

Variable d'interaction : la variable d'interaction entre les performances initiales des élèves en code et le rendement effectif n'est pas significative.

Dans le modèle 5, la performance initiale des élèves en code, la catégorie socioprofessionnelle des parents, l'âge, le rythme scolaire de la semaine et la variable rend_eff_29 influencent la performance finale des élèves en code. 